\title{
Localised Mass-forming Amyloidosis of the Genitourinary Tract Mimics Urothelial Carcinoma: Two Cases of Mistaken Identity
}

\author{
(D) Pranav Sharma1,2, (D) Aiyapa Aruna Ajjikuttira1, (D) Handoo Rhee1,2, (D) Darius Ashrafi2,3, (D) Matthew John Roberts²,4, \\ (D) Eric Chung 1,2
}

1 Princess Alexandra Hospital, Clinic of Urology, Brisbane, Australia

2University of Queensland Faculty of Medicine, Brisbane, Australia

${ }^{3}$ Bankstown-Lidcombe Hospital, Clinic of Urology, Sydney, Australia

${ }^{4}$ Royal Brisbane and Women's Hospital, Clinic of Urology, Brisbane, Australia

\begin{abstract}
Amyloidosis of the lower genitourinary tract is an extremely rare condition and often mimics urothelial malignancy. In this paper, we report two cases of ureteral and urethral amyloidosis that initially presented as urothelial carcinoma and were successfully treated with laparoscopic nephroureterectomy and trans-urethral resection, respectively. This disease, with its various atypical presentations, presents a diagnostic challenge to urologists. Awareness is important as excision can be curative, and presence of systemic disease must be excluded.
\end{abstract}

Keywords: Amyloidosis, ureter, urethra

\section{Introduction}

Amyloidosis is a pathological process involving extracellular deposition of abnormal protein fibrils within the body. Localised amyloidosis of the genitourinary tract is a rare entity and may masquerade as urothelial carcinoma clinically, radiologically and endoscopically. In this report, we describe our experience with isolated mass-forming amyloidosis of the ureter and urethra.

\section{Case 1}

A 57-year-old woman presented with left-sided flank pain of 2 months duration. She denied visible haematuria and other lower urinary tract symptoms. She was a non-smoker and had no history of urolithiasis. Her estimated glomerular filtration rate was $>90 \mathrm{~mL} / \mathrm{min} / 1.73 \mathrm{~m}^{2}$ with a serum creatinine of $53 \mathrm{mg} /$ $\mathrm{dL}$. Urine culture was sterile.

An initial computed tomography intravenous pyelogram demonstrated a $17 \times 8 \mathrm{~mm}^{2}$ contrast-enhancing lesion of the distal left ureter, raising suspicion for a urothelial tumour (Figure 1). Urine cytology, however, was negative for high-grade urothelial cancer.
An attempt at endoscopic biopsy of the lesion was unsuccessful because of a dense distal ureteric stricture. The patient therefore underwent laparoscopic left nephroureterectomy on the basis of a clinical and radiological suspicion for a primary urothelial malignancy. Histopathology confirmed mass-forming amyloidosis in the ureter without evidence of neoplasia. There was no amyloid deposition in the kidney. Immunohistochemistry for light chains and amyloid A did not contribute in the typing of ureteric amyloid.

This patient was followed up 12 months later with a negative result for systemic amyloidosis. Her kidney function is stable, and she remains disease-free with no recurrence.

\section{Case 2}

A 23-year-old man presented with intermittent urethral bleeding and haematospermia. He described obstructive voiding symptoms, including a poor stream and spraying on voiding. On examination, he had a palpable urethral mass at the level of the coronal sulcus, presumed to be a urethral malignancy. He was a non-smoker with a history of treated chlamydial urethritis.

Correspondence: Pranav Sharma MD, Princess Alexandra Hospital, Clinic of Urology and University of Queensland Faculty of Medicine, Brisbane, Australia Phone: +61 $731762111 \quad$ E-mail: pranavs041@gmail.com 0RCID-ID: orcid.org/0000-0002-3743-2953

Received: 26.12 .2020 Accepted: 10.03.2021

Cite this article as: Sharma $\mathrm{P}$, Ajjikuttira AA, Rhee $H$, Ashrafi D, Roberts MJ, Chung E. Localised Mass-forming Amyloidosis of the Genitourinary Tract Mimics Urothelial Carcinoma: Two Cases of Mistaken Identity. J Urol Surg 2021;8(2):142-144.

${ }^{\circ}$ Copyright 2021 by the Association of Urological Surgery / Journal of Urological Surgery published by Galenos Publishing House. 
A papillary lesion was visualised in the anterior urethra with a 22-Fr rigid cystoscope. The mass was completely avulsed with passage of the scope, followed by completion cold-cup biopsy and Bugbee diathermy haemostasis. Histopathology showed mass-forming amyloidosis containing a mixed infiltrate of plasma cells and lymphocytes, in keeping with localised amyloidosis (Figure 2).

The patient was examined 12 months later, with no symptoms suggestive of recurrence and a negative result for systemic amyloidosis. Written and verbal informed consent was obtained from both patients to report their case and publish related clinical images.

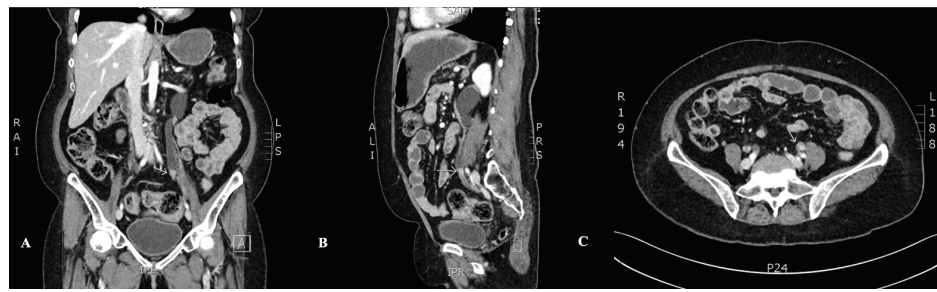

Figure 1. Coronal (A), sagittal (B) and axial (C) views on abdominal computed tomography demonstrating a contrast-enhancing soft tissue mass within the distal left ureter with upstream hydroureteronephrosis

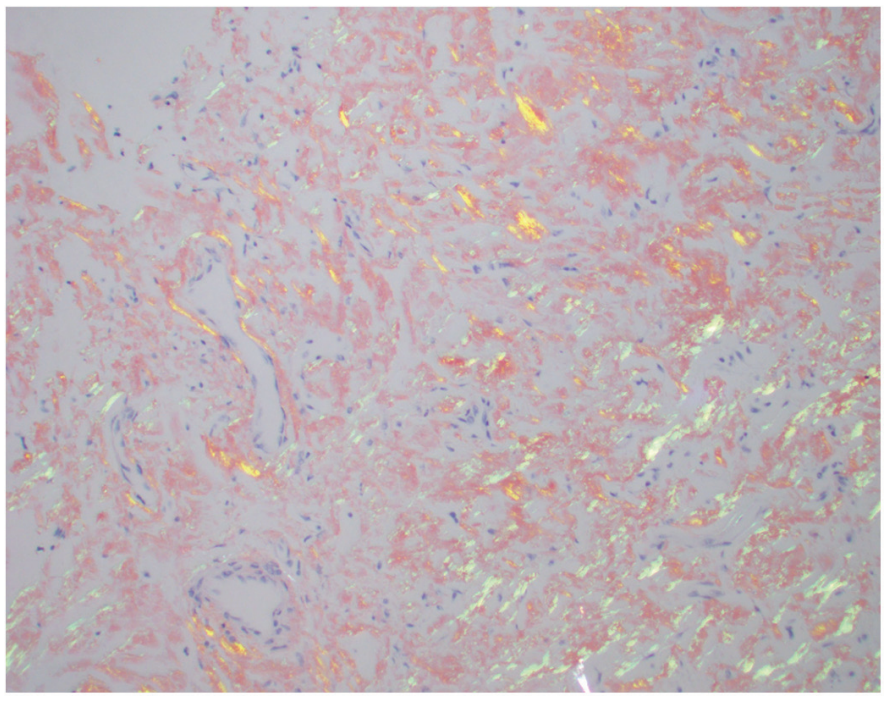

Figure 2. Histopathology slide of the urethral mass with Congo red stain displaying an apple-green birefringence (bottom right of image)

\section{Discussion}

Amyloidosis can be systemic (multiorgan involvement) or localised (limited to one organ). Within the genitourinary tract, amyloidosis more commonly involves the bladder and rarely the ureter, renal pelvis or urethra (1). Herein, we presented two rare cases of ureteral and urethral amyloidosis masquerading as urothelial malignancy. Management included nephroureterectomy and trans-urethral resection, respectively, with no disease recurrence at 12 months.

Amyloidosis results from extracellular deposition of abnormal proteins in healthy tissue. These mis-folded fibrils aggregate into beta-pleated sheets that disrupt normal function. Primary amyloidosis (AL) arises from abnormal plasma cells producing excessive light chains that eventually deposit into tissues. Secondary amyloidosis (AA) is typically seen in inflammatory and autoimmune conditions such as Chron's disease, in which serum amyloid A protein (SAA), an acute phase reactant, is persistently elevated. SAA is amyloidogenic at chronically high levels, and up to $10 \%$ of patients eventually develop AA-type amyloidosis (2).

Diagnosis is made with biopsy. Staining with Congo red dye, which displays an apple-green birefringence under polarised light, confirms the diagnosis (3). Electron microscopy shows non-branching rigid 8-12 mm fibrils (3). Immunohistochemistry can be used for typing (e.g. kappa or lambda light chains).

\section{Ureter}

The deposition of amyloid in the ureter is uncommon and usually unifocal, often involving the distal ureter (4). Patients usually present with haematuria and symptoms of ureteric obstruction such as flank pain. There is predilection in women (female-tomale ratio of 3:2), and the left ureter is more commonly affected than the right (5). Synchronous or metachronous bilateral involvement is seen in up to $12.7 \%$ of cases and may present with obstructive uropathy and renal failure (5).

Radiological findings include diffuse or focal wall thickening, intra-ureteral filling defects and irregular narrowing and stricturing with subsequent upstream hydronephrosis $(4,6)$. These imaging findings are indistinguishable from urothelial neoplasia; thus, biopsy is recommended. Amyloidosis, along with tuberculosis and schistosomiasis, should be considered in the non-neoplastic differential diagnosis (7).

Historically, amyloidosis of the ureter has been treated with nephroureterectomy (6). However, if amyloidosis can be diagnosed preoperatively with biopsy, one of two nephronsparing conservative approaches can be used. One is segmental resection of the ureter including resection with end-to-end anastomosis (uretero-ureterostomy), ureteral reimplantation (ureteroneocystostomy), auto-transplantation and ileal ureter replacement after confirming the pathology by frozen section (6). Another treatment includes ureteric stenting with occlusive dressing using dimethyl sulfoxide for 6 months, leading to complete resolution of the lesion $(4,6)$.

A radical approach was taken in Case 1, as the surgeons were unable to obtain an endoscopic biopsy safely. The suspicion was that it was an urothelial malignancy, until proven otherwise; 
therefore, a nephroureterectomy was performed. Overtreatment would have been avoided if confirmatory biopsy was feasible.

\section{Urethra}

The urethra is a rare site for amyloidosis and has mainly been reported in men (5). Patients with urethral amyloidosis usually present with urethral bleeding, dysuria or obstructive voiding symptoms. Lesions are most commonly unifocal, and any portion of the urethra may be affected. Imaging findings include areas of hypointense urethral and periurethral mucosa on T2weighted magnetic resonance imaging (MRI) and urethral foci of increased echogenicity with posterior shadowing on penile ultrasonography (4).

Because the differential diagnosis includes urothelial cancer, urethroscopy with trans-urethral resection is recommended. Clinically, a mass, mucosal friability and/or stricturing are seen with urethroscopy. Differential diagnosis includes structural abnormalities such as urethral valves, urethral cysts or fibroepithelial polyps, which can present as stricture or mass (2). Other benign conditions in the differential diagnosis include nephrogenic adenoma, malakoplakia, condyloma and ectopic prostatic tissue. In women, urethral caruncles and urethral diverticula can present as a urethral mass $(2,4)$.

In almost all published cases, the condition appear to be selflimiting and no specific treatment is required, other than excision of the lesion and appropriate management of any stricture formation (8).

\section{Systemic Workup}

The most common site for systemic amyloidosis includes the kidneys, heart, nerves and liver (9). In our institute, we routinely perform kidney and liver function tests as well as serum and urine protein electrophoresis, urine Bence-Jones proteins and serum free light chains to exclude systemic disease. Cardiac biomarkers such as troponin and B-type natriuretic peptide are also measured, and a baseline electrocardiogram is obtained. If cardiac amyloid is suspected, a thoracic echocardiography and cardiac MRI is suggested. Nerve conduction studies are performed if peripheral neuropathy is present. A bone marrow biopsy may also be required to confirm the presence of abnormal plasma cells.

Treatment of systemic disease may require a combination of chemotherapy, corticosteroids and autologous stem cell transplant (9).

\section{Conclusion}

We presented two rare cases of ureteral and urethral massforming amyloidosis that presented as urothelial malignancy.
Preoperative biopsy is recommended for ureteral lesions to avoid radical surgery and to spare the kidneys. Urethral amyloidosis can be managed with trans-urethral resection with good outcomes. Referral to a haematologist should be made for evaluation of systemic disease.

\section{Ethics}

Informed Consent: Written and verbal informed consent was obtained from both patients to report their case and publish related clinical images.

Peer-review: Externally peer-reviewed.

\section{Authorship Contributions}

Surgical and medical practices: H.R., M.J.R., E.C., Concept: E.C., Design: P.S., Data Collection or Processing: P.S., Analysis or Interpretation: P.S., Literature Search: P.S., Writing: P.S., A.A.A., H.R., D.A., M.J.R., E.C.

Conflict of Interest: No conflict of interest was declared by the authors.

Financial Disclosure: The authors declared that this study received no financial support.

\section{References}

1. Chitale $S$, Morsey M, Peat D, Webb R. Amyloidosis of the lower genitourinary tract: A review. EAU-EBU Update Series 2007;5:70-76.

2. Isaacson $A L$, Dahmoush $\mathrm{L}$, Robinson RA. Primary localised amyloidosis presenting as urethral stricture: An important differential diagnosis on frozen section. Human Pathology: Case Reports 2020;20:200365.

3. Paner GP. Amyloidosis. American Urological Association Web site. https:// www.auanet.org/education/auauniversity/education-products-andresources/pathology-for-urologists/urinary-bladder/non-neoplasticlesions/amyloidosis. Published 2012. Accessed November 28, 2020.

4. Kawashima A, Alleman WG, Takahashi N, Kim B, King BF Jr, LeRoy AJ. Imaging evaluation of amyloidosis of the urinary tract and retroperitoneum. Radiographics 2011;31:1569-1582.

5. Park HS, Cho HY. Amyloidosis of the urogenital system. Korean J Urol Oncol 2010;8:59-64.

6. Tsujioka Y, Jinzaki M, Tanimoto A, Nakagawa K, Akita H, Kikuchi E, Okuda S, Mikami S, Oya M, Kuribayashi S. Radiological findings of primary localized amyloidosis of the ureter. J Magn Reson Imaging 2012;35:431-435.

7. Mark IR, Goodlad J, Lloyd-Davies RW. Localized amyloidosis of the genitourinary tract. J R Soc Med 1995;88:320-324.

8. Mangera A, Linton KD, Fernando M, Channer J, Chapple CR. What is the evidence for the management of urethral amyloidosis? A systematic review of the literature. BJU Int 2012;109:1858-1861.

9. Amyloidosis: A guide for patients and families. Leukemia Foundation Web site. https://www.leukaemia.org.au/wp-content/uploads/2020/06/ Amyloidosis-July-2017_PDF.pdf. Updated July 2017. Accessed November 20, 2020. 